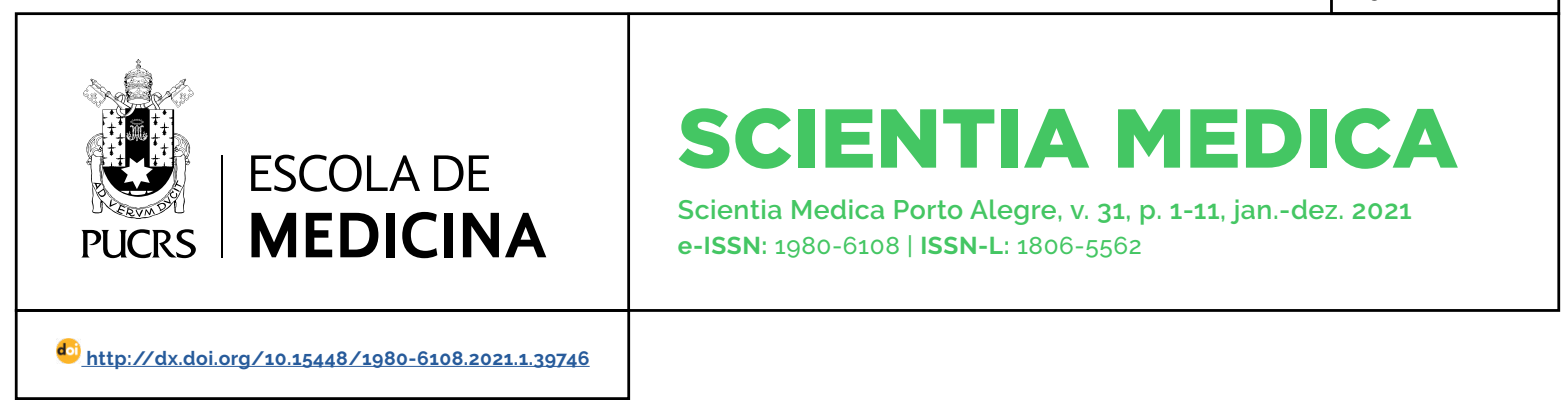

ARTIGO ORIGINAL

\title{
Crescimento pôndero-estatural de crianças e adolescentes submetidos à adenoamigdalectomia
}

\author{
Weight and height growth of children and adolescents after adenoton sillectomy
}

\section{André Costa Pinto Ribeiro 1 \\ orcid.org/0000-0001-9955-6073 \\ andrecpribeiro@yahoo.com.br}

\section{Tarssius Capelo Candido ${ }^{1}$}

orcid.org/0000-0003-4555-5117 tarssiuscapelo@gmail.com

\section{Pedro Henrique Almeida Nascimento ${ }^{1}$} orcid.org/0000-0002-1866-5938 pedroh_an@hotmail.com

\section{Paula Ferraz Rodrigues ${ }^{1}$} orcid.org/0000-0002-0792-2337 paulalgp@hotmail.com

\section{Wilson Benini Guércio ${ }^{1}$ orcid.org/0000-0002-8764-767X beniniguercio@yahoo.com.br}

Recebido em: 13 dez. 2020 Aprovado em: 15 fev. 2021. Publicado em: 13 abril. 2021.

\section{Resumo}

Objetivo: documentar de forma sistemática o padrão de desenvolvimento pôndero-estatural de pacientes submetidos à adenoamigdalectomia.

Métodos: coleta de dados secundários dos prontuários de pacientes atendidos no ambulatório de Otorrinolaringologia Pediátrica, antes e depois da cirurgia de adenoamigdalectomia.

Resultados: de forma individual, os pacientes apresentaram elevação no escore $\mathrm{Z}$ e percentis das variáveis ao peso e a altura no periodo entre as análises. Especificamente em relação ao peso, a média antes do procedimento e quatro meses depois do procedimento foi, respectivamente, de 29,1 kg e 32,8 kg; no que diz respeito à altura, a média foi de 1,22 $\mathrm{m}$ e 1,25 $\mathrm{m}$, respectivamente. Ao aplicar o Teste T de Student foi possivel notar significância estatística para ambas as variáveis em estudo. Aspecto não percebido ao avaliar os indivíduos reunidos em grupos etários (pré-escolares, escolares e adolescentes).

Conclusões: as crianças submetidas à adenoamigdalectomia apresentaram ganho pôndero-estaturalapós a cirurgia. A atuação cirúrgica diante do diagnóstico da hipertrofia e da hiperplasia das amígdalas e tonsila faringea deve ser precoce. desde que haja indicação formal, a fim de evitar a manutenção do atraso no crescimento nesses pacientes.

Palavras-chave: adenoidectomia, adenoamigdalectomia, tonsilectomia, crescimento pôndero-estatural, avaliação de resultado de intervenções terapêuticas.

\section{Abstract}

Aims: to systematically document weight and height development in children after adenotonsillectomy.

Methods: analysis of secondary data from the Pediatric Otorhinolaryngology clinic patient's medical records before and after adenotonsillectomy.

Results: regarding weight, the average before the procedure and four months and the average after the procedure were, respectively, $29.1 \mathrm{~kg}$ and $32.8 \mathrm{~kg}$; as regards height, the averages were $1.22 \mathrm{~m}$ and $1.25 \mathrm{~m}$, respectively. By applying the Teste T de Student it was possible to notice statistical significance for both variables under study. Conclusions: children exhibited statistically significant weight and height gain after surgery. Surgical treatment should be performed early after the diagnosis of pharyngeal tonsil and tonsils hypertrophy and hyperplasia provided in case of a formal indication in order to avoid the maintenance growth retardation in these patients.

Keywords: adenoidectomy, adenotonsillectomy, tonsillectomy, growth weight pond, evaluation of results of the trapeutic interventions. 


\section{Introdução}

O anel de Waldeyer compreende um conjunto de estruturas linfoepiteliais que se agrupam em formato circular na porção superior do trato aereodigestivo e que possuem função de proteção imunológica, apresentando papel importante na defesa contra infecções, fornecendo resposta a antígenos exógenos provenientes do ar e dos alimentos. Seus componentes, classificados de acordo com sua localização, são: um par de tonsilas palatinas (também nomeadas de amigdalas palatinas ou amigdalas), a tonsila faringea (tonsila nasofaringea ou adenoide), um par de tonsilas tubárias, tonsilas linguais e pequenas coleções na parede da faringe. As tonsilas palatinas e a tonsila faringea possuem maior dimensão e são consideradas as mais ativas (1).

As tonsilas estão presentes já ao nascimento; fisiologicamente aumentam durante o periodo pré-escolar, quando ocorre hipertrofia intensa, podendo proporcionar obstrução das vias áreas superiores (2). O diagnóstico da hipertrofia das amígdalas pode ser realizado durante o exame fisico por meio da oroscopia; já a tonsila faringea através de radiografia de cavum ou por meio da fibronasoendoscopia (3).

A redução da dimensão da via área na criança pode ocasionar problemas irreversiveis, estando diretamente correlacionada ao prolongar do comprometimento do padrão respiratório. A adenoamigdalectomia é um dos procedimentos cirúrgicos mais realizados no mundo, sendo a principal causa de internação hospitalar cirúrgica em crianças e adolescentes no Brasil, além de alcançar 500 mil cirurgias por ano nos Estados Unidos da América (2, 4, 5, 6, 7, 8).

As principais indicações cirúrgicas são: hipertrofia adenoamigdaliana com obstrução das vias aéreas, sindrome da apneia obstrutiva do sono, distúrbios de deglutição e fonação, tonsilites de caráter recorrente e tonsilite crônica ou tonsilite caseosa. Quando a cirurgia é postergada ou não é efetuada, os pacientes podem ter comprome- timentos como desatenção, sonolência diurna, enurese noturna e complicações locorregionais $(3,6,7,9,10)$.

Apresentam-se com destaque as alterações no desenvolvimento pôndero-estatural de pacientes portadores de hipertrofia das tonsilas, cujo retardo do crescimento abrange prevalência de até $46 \%$ dos pacientes. A possivel explicação para este déficit de crescimento advém da combinação de diversos fatores: modificações anatômicas, desenvolvimento da sindrome da apneia obstrutiva do sono, alteração hormonal e infecções recorrentes $(5,7,11)$.

A exérese das tonsilas pode ocasionar melhora no desenvolvimento pôndero-estatural, com retomada do crescimento. Já foi demonstrado que a taxa de crescimento após a realização de adenoamigdalectomia é estatisticamente significativa, com um aumento de $34 \%$ e $57 \%$ nas medidas de altura e peso, respectivamente $(5,11,12)$.

No serviço de otorrinolaringologia onde fora desenvolvido a pesquisa é recorrente o atendimento de crianças que se encontram abaixo dos escores ideais nas curvas de peso e altura e apresentam hipertrofia e hiperplasia das tonsilas e com indicação cirúrgica. Sendo um serviço de residência médica, deve-se atentar que é um momento de treinamento em serviço para o futuro médico especialista. É o momento em que o médico irá obter progressiva responsabilidade profissional, incremento da capacidade de iniciativa, julgamento e avaliação, aquisição de preceitos e normas éticas, bem como espirito critico (13, 14). Dessa forma, é fundamental apresentar e reforçar a necessidades de condutas acadêmicas que são realizadas, refutando aquelas que não são cientificamente comprovadas e reforçando as comprovadas de maneira expositiva sempre que possivel.

A fim de alcançar esse último objetivo, aliado ao fato de que até o momento não se conhece de forma sistemática os padrões de desenvolvimento pôndero-estatural desses pacientes posteriormente à cirurgia, torna-se relevante analisar as 
diferenças nos padrões dessas crianças a fim de detectar e de demonstrar os benefícios do procedimento em crianças com déficit de crescimento. O objetivo do estudo foi documentar de forma sistemática os padrões de desenvolvimento pôndero-estatural de crianças e de adolescentes submetidos à adenoamigdalectomia.

\section{Métodos}

A pesquisa realizada foi classificada quanto à área da ciência aplicada, à natureza original, ao objetivo exploratório e à abordagem quantitativa. Foi realizado um estudo de coorte retrospectiva, com seleção aleatória da amostra, no qual foram analisadas as medidas antropométricas (peso e altura) antes e depois da cirurgia de adenoamigdalectomia, tendo, assim, caráter evolutivo em relação ao estado do paciente, apresentando, dessa forma, caracteristica prospectiva para cada indivíduo.

A coleta dos dados ocorreu posteriormente, tendo como fonte informações secundárias dos prontuários de pacientes atendidos no ambulatório de otorrinolaringologia pediátrica do Hospital Universitário da Universidade Federal de Juiz de Fora. Ressalta-se que, a aleatorização decorreu da seleção dos 30 primeiros prontuários selecionados e que satisfaziam os critérios de inclusão.

Os critérios de inclusão da amostra foram: pacientes com a idade entre três e16 anos, atendidos no ambulatório de otorrinolaringologia pediátrica, com indicação de adenoamigdalectomia por hipertrofia, no período de março a dezembro de 2018 , submetidos a medidas de avaliação pôndero-estatural na data da cirurgia. Os pacientes possuiam hipertrofia das amigdalas de grau III ou grau IV. Em relação à tonsila faríngea, obstrução superior a 70\% da cavidade nasal posterior. Não foi incluído nenhum paciente com diagnóstico estabelecido para patologias sistêmicas associadas, alterações neurológicas ou do esqueleto craniofacial. Foram excluidos aqueles pacientes que possuíam dados incompletos no sistema de prontuário.
Essas medidas foram feitas em dois momentos: imediatamente anterior ao procedimento cirúrgico e após quatro meses da adenoamigdalectomia. A diferença entre as medidas foi comparada com a média de crescimento e de ganho de peso na população normal com a mesma idade no mesmo intervalo de tempo $(15,16)$.

As medidas antropométricas de todos os participantes da pesquisa foram realizadas por um único pesquisador treinado previamente. A aferição dos dados foi obtida pela utilização de uma única balança plataforma com antropômetro da marca Wely ${ }^{\circledR}$, modelo $110 \mathrm{CH}$, sempre com a calibração em dia, localizada em local de superficie plana, distante da parede.

As crianças e os adolescentes foram posicionados de costas para a balança, com os pés juntos e os braços ao longo do corpo, vestindo apenas peça intima e na presença de seu responsável (pais ou responsável legal). Com auxilio do antropômetro vertical foi realizada a determinação da altura. O índice de massa corpórea (IMC) foi calculado no momento das aferições, bem como a inserção dos dados nas planilhas de controle.

Os participantes foram agrupados de acordo com a nomenclatura estabelecida pela Sociedade Brasileira de Pediatria, que define como periodo pré-escolar a idade de dois a seis anos; periodo escolar aquele que compreende a faixa etária de sete aos dez anos; e, adolescência, período da vida que se inicia aos dez anos de idade e se prolonga até os 20 anos (17).

Tratando-se de um estudo longitudinal com análise de uma mesma amostra em dois momentos distintos, foi realizada a avaliação das médias e analisada a possibilidade de correlação com resultados encontrados em âmbito individual do e agrupado sob avaliação estatística por meio do Teste T de Student. A análise de dados foi feita no programa R Studio e confirmada por meio do site Social Science Statistics. A apreciação dos resultados obtidos respeitou as seguintes normas: intervalo de confiança de 95\% e p-valor 
menor do que 0,05 (16). Com intuito comparativo, foram utilizados os referencias antropométricos em termos de percentis e escore Z pré-estabelecidos pela Organização Mundial de Saúde, que determina as curvas de crescimento. Sendo assim avaliados antes e posteriormente a quatro meses do procedimento cirúrgico.

Uma vez que a pesquisa se desenvolveu através de dados secundários, não foi necessária a utilização do Termo de Consentimento Livre Esclarecido e foi devidamente submetida e aprovada pelo Comitê de Ética em Pesquisa da instituição do Hospital Universitário da Universidade Federal de Juiz de Fora, segundo parecer de $\mathrm{n}^{\circ}$ 3.434.372.

\section{Resultados}

Foram estudados 29 indivíduos (16 meninas e 13 meninos), entre três e 13 anos idade, sendo 15 pré-escolares, sete escolares e sete adolescentes. Em relação ao peso, a média antes do procedimento e quatro meses depois do procedimento foi, respectivamente, de 29,1 $\pm 17,4$ $\mathrm{kg}$ e $32,8 \pm 18,7 \mathrm{~kg}(\mathrm{p}<0,0001)$. No que diz respeito à altura, a média antes do procedimento cirúrgico foi de $122 \pm 27,1 \mathrm{~cm}$ e quatro meses depois da cirurgia foi $125 \pm 25,4$ ( $p<0,0001$ ). Ao avaliar os dados de forma combinada, a fim de obter o IMC, ocorreu elevação nos índices obtidos após o procedimento. Em comparação com os índices obtidos antes do procedimento, de 17 $\pm 4,6$ para $19,0 \pm 5,1$ sendo percebida a elevação em 20 participantes da pesquisa.

No que diz respeito à altura, que possui referencial para inclusão dos adolescentes, notou-se o incremento nos dados individuais de Z escore e percentil de $21 \mathrm{em} 29$ dos individuos. Dentre os individuos que puderam ser classificados quanto ao percentil e ao $Z$ escore, 20 dos 22 participantes apresentaram alterações positivas em seus valores, o que indica crescimento, entre as medições.

Ao realizar a análise por grupos etários percebeu-se que, em relação ao peso, a média antes do procedimento e quatro meses depois do procedimento foi, respectivamente, de 10,9 $\pm 9,5 \mathrm{~kg} \mathrm{e}$ $12,7 \pm 10,5 \mathrm{~kg}$ ( $p=0,27$ ) em pré-escolares; de 14,9 $\pm 12,7$

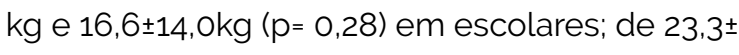
$22,9 \mathrm{~kg}$ e $27,2 \pm 23,5 \mathrm{~kg}(\mathrm{p}=0,33)$ em adolescentes.

Ainda em relação à análise dos grupos etários, a variável altura antes do procedimento e quatro meses depois do procedimento apresentou os seguintes resultados: 104,7 $\pm 10,4 \mathrm{~cm}$ e 108,1 $\pm 11,1$ $\mathrm{cm}(\mathrm{p}=0,27)$ em pré-escolares; $119,6 \pm 10,1 \mathrm{~cm} \mathrm{e}$ $123,9 \pm 8,3 \mathrm{~cm}(p=0,28)$ em escolares; e 149,5 $\pm 7,8$ $\mathrm{cm}$ e 151,8 $7,3 \mathrm{~cm}(p=0,33)$ em adolescentes.

Os dados compilados no pré e no pós-operatório são apresentados na Tabela 1. 
TABELA 1 - Dados sobre peso e altura individualizados dos participantes mensurados na data da cirurgia (pré-operatório) e quatro meses após a cirurgia (pós-operatório)

\begin{tabular}{|c|c|c|c|c|c|c|c|c|c|c|c|c|c|c|}
\hline Indivíduo & Sexo & $\begin{array}{l}\text { Idade pós } \\
\text { procedimento }\end{array}$ & Peso Pré & $\begin{array}{l}\text { Percentil } \\
\text { Pré }\end{array}$ & $\begin{array}{l}Z \text { escore } \\
\text { Pré }\end{array}$ & Peso Pós & $\begin{array}{l}\text { Percentil } \\
\text { Pós }\end{array}$ & $\begin{array}{l}Z \text { escore } \\
\text { Pós }\end{array}$ & $\begin{array}{l}\text { Altura } \\
\text { Pré }\end{array}$ & $\begin{array}{l}\text { Percentil } \\
\text { Pré }\end{array}$ & $\begin{array}{l}Z \text { escore } \\
\text { Pré }\end{array}$ & $\begin{array}{l}\text { Altura } \\
\text { Pós }\end{array}$ & $\begin{array}{l}\text { Percentil } \\
\text { Pós }\end{array}$ & $\begin{array}{l}Z \text { escore } \\
\text { Pós }\end{array}$ \\
\hline \multicolumn{15}{|l|}{ Pré Escolar } \\
\hline 24 & M & 3 anos e 5 meses & 17,5 & $4^{\circ}(93,3)$ & 1,5 & 17,1 & $4^{\circ}(82,3)$ & 0,93 & 94 & $1^{\circ}(21,70$ & $-0,78$ & 96 & $1^{\circ}(19,1)$ & $-0,88$ \\
\hline 25 & $\mathrm{~F}$ & 3 anos e 9 meses & 13,8 & $2^{\circ}(39,1)$ & $-0,55$ & 15,5 & $2^{\circ}(48,7)$ & $-0,03$ & 93 & $1^{\circ}(8,4)$ & $-1,38$ & 95 & $1^{\circ}(7,4)$ & $-1,45$ \\
\hline 26 & M & 3 anos e 7 meses & 14,0 & $2^{\circ}(29,3)$ & -0.54 & 16,0 & $3^{\circ}(57,3)$ & 0,18 & 98 & $2^{\circ}(45,3)$ & $-0,12$ & 100 & $2^{\circ}(41,1)$ & $-0,22$ \\
\hline 2 & F & 3 anos e 11 meses & 16,5 & $3^{\circ}(72,6)$ & 0,6 & 20,2 & $4^{\circ}(95)$ & 1,65 & 100 & $3^{\circ}(51,7)$ & 0,4 & 102 & $2^{\circ}(47,2)$ & $-0,7$ \\
\hline 22 & M & 4 anos e 5 meses & 12,5 & $1^{\circ}(1,2)$ & $-2,26$ & 13,4 & $1^{\circ}(2,4)$ & $-1,98$ & 92 & $1^{\circ}(0,2)$ & $-2,89$ & 95 & $1^{\circ}(0,4)$ & $-2,62$ \\
\hline 9 & $\mathrm{~F}$ & 5 anos e 4 meses & 19,1 & $3^{\circ}(60,8)$ & 0,27 & 20,7 & $3^{\circ}(73,1)$ & 0,62 & 113 & $4^{\circ}(75,1)$ & 0,68 & 118 & $4^{\circ}(90,8)$ & 1,33 \\
\hline 10 & $\mathrm{~F}$ & 5 anos e 4 meses & 18,0 & $2^{\circ}(44,6)$ & $-0,14$ & 20,3 & $3^{\circ}(68,2)$ & 0,47 & 110 & $1^{\circ}(3,2)$ & $-1,85$ & 111 & $2^{\circ}(45,2)$ & $-0,12$ \\
\hline 11 & M & 5 anos e 5 meses & 16,5 & $1^{\circ}(17,1)$ & $-0,95$ & 19,2 & $2^{\circ}(47,6)$ & $-0,6$ & 106 & $1^{\circ}(15,6)$ & $-1,01$ & 110 & $2^{\circ}(28,0)$ & $-0,58$ \\
\hline 15 & $\mathrm{~F}$ & 5 anos e 9 meses & 26,0 & $4^{\circ}(97,8)$ & 2,01 & 35,9 & $4^{\circ}(100)$ & 3,66 & 120 & $4^{\circ}(95,4)$ & 1,69 & 124 & $4^{\circ}(98,0)$ & 2,05 \\
\hline 17 & F & 5 anos e 9 meses & 37,8 & $4^{\circ}(100)$ & 4,29 & 39.9 & $4^{\circ}(100)$ & 4.33 & 121 & $4^{\circ}(96,3)$ & 1,79 & 124 & $4^{\circ}(97.5)$ & 1,96 \\
\hline 1 & $\mathrm{~F}$ & 5 anos e 10 meses & 15,0 & $1^{\circ}(3,8)$ & $-1,77$ & 18,6 & $2^{\circ}(33,1)$ & $-0,44$ & 105 & $1^{\circ}(7,3)$ & $-1,45$ & 114 & $2^{\circ}(48,7)$ & $-0,3$ \\
\hline \multicolumn{15}{|l|}{ Escolar } \\
\hline 21 & M & 6 anos e 1 mês & 20,6 & $3^{\circ}(57,2)$ & 0,19 & 22,5 & $3^{\circ}(71,2)$ & 0,56 & 120 & $4^{\circ}(85,6)$ & 1,06 & 122 & $4^{\circ}(85,1)$ & 1,04 \\
\hline 14 & M & 6 anos e 1 mês & 19,7 & $2^{\circ}(45,4)$ & $-0,12$ & 22,0 & $3^{\circ}(66,4)$ & 0,42 & 117 & $3^{\circ}(68,9)$ & 0,49 & 120 & $4^{\circ}(75,2)$ & 0,68 \\
\hline 4 & M & 6 anos e 2 meses & 26,0 & $4^{\circ}(96,8)$ & 1,85 & 28,3 & $4^{\circ}(98,4)$ & 2,14 & 124 & $4^{\circ}(96,9)$ & 1,86 & 127 & $4^{\circ}(97,8)$ & 2,01 \\
\hline
\end{tabular}




\begin{tabular}{|c|c|c|c|c|c|c|c|c|c|c|c|c|c|c|}
\hline Indivíduo & Sexo & $\begin{array}{l}\text { Idade pós } \\
\text { procedimento }\end{array}$ & Peso Pré & $\begin{array}{l}\text { Percentil } \\
\text { Pré }\end{array}$ & $\begin{array}{l}Z \text { escore } \\
\text { Pré }\end{array}$ & Peso Pós & $\begin{array}{l}\text { Percentil } \\
\text { Pós }\end{array}$ & $\begin{array}{l}Z \text { escore } \\
\text { Pós }\end{array}$ & $\begin{array}{l}\text { Altura } \\
\text { Pré }\end{array}$ & $\begin{array}{l}\text { Percentil } \\
\text { Pré }\end{array}$ & $\begin{array}{l}Z \text { escore } \\
\text { Pré }\end{array}$ & $\begin{array}{l}\text { Altura } \\
\text { Pós }\end{array}$ & $\begin{array}{l}\text { Percentil } \\
\text { Pós }\end{array}$ & $\begin{array}{l}Z \text { escore } \\
\text { Pós }\end{array}$ \\
\hline \multicolumn{15}{|l|}{ Escolar } \\
\hline 8 & M & 6 anos e 2 meses & 15,2 & $1^{\circ}(1,3)$ & $-2,22$ & 17,8 & $1^{\circ}(10,7)$ & $-1,24$ & 105 & $2^{\circ}(2,1)$ & $-2,6$ & 111 & $1^{\circ}(11,3)$ & $-1,21$ \\
\hline 19 & $\mathrm{~F}$ & 6 anos e 7 meses & 17,5 & $1^{\circ}(11,7)$ & $-1,19$ & 19.5 & $2^{\circ}(25.5)$ & $-0,66$ & 115 & $2^{\circ}(36,6)$ & $-0,35$ & 117 & $2^{\circ}(37.5)$ & $-0,32$ \\
\hline 30 & $\mathrm{~F}$ & 7 anos e 1 mês & 18,5 & $1^{\circ}(12,8)$ & $-1,14$ & 19,7 & $1^{\circ}(17,5)$ & $-0,93$ & 102 & $1^{\circ}(0,1)$ & $-3,26$ & 114 & $1^{\circ}(8,6)$ & $-1,36$ \\
\hline 3 & M & 7 anos e 2 meses & 26,0 & $4^{\circ}(83,8)$ & 0,99 & 33.5 & $4^{\circ}(99,1)$ & 2,37 & 120 & $2^{\circ}(41,7)$ & $-0,21$ & 129 & $4^{\circ}(86,9)$ & 1,12 \\
\hline 20 & M & 7 anos e 3 meses & 22,7 & $2^{\circ}(48,8)$ & $-0,03$ & 25,8 & $3^{\circ}(73.3)$ & 0,62 & 121 & $2^{\circ}(46,2)$ & $-0,10$ & 123 & $2^{\circ}(47,1)$ & $-0,07$ \\
\hline 7 & M & 8 anos e 2 meses & 46,8 & $4^{\circ}(100)$ & 3.95 & 53,4 & $4^{\circ}(100)$ & 4,48 & 136 & $4^{\circ}(95,3)$ & 1,68 & 139 & $4^{\circ}(96,8)$ & 1,85 \\
\hline 27 & $\mathrm{~F}$ & 8 anos e 4 meses & 28,0 & $2^{\circ}(73,9)$ & 0,64 & 34.5 & $4^{\circ}(94)$ & 1,56 & 124 & $2^{\circ}(31,4)$ & $-0,48$ & 128 & $2^{\circ}(44,7)$ & $-0,13$ \\
\hline 16 & $\mathrm{~F}$ & 9 anos e 4 meses & 35,0 & $4^{\circ}(89,2)$ & 1,24 & 37,6 & $4^{\circ}(90,8)$ & 1,33 & 132 & $2^{\circ}(48,4)$ & $-0,04$ & 133 & $2^{\circ}(38,7)$ & $-0,29$ \\
\hline \multicolumn{15}{|c|}{ Adolescência } \\
\hline 5 & $\mathrm{~F}$ & 10 anos e 9 meses & 36,1 & ND & ND & 40,8 & ND & ND & 140 & $2^{\circ}(39,4)$ & $-2,7$ & 146 & $3^{\circ}(62,5)$ & 0,32 \\
\hline 6 & $\mathrm{~F}$ & 11 anos e 9 meses & 36,2 & ND & ND & 38,8 & ND & ND & 143 & $1^{\circ}(23,2)$ & $-0,73$ & 145 & $1^{\circ}(23,0)$ & $-0,74$ \\
\hline 23 & M & 11 anos e 9 meses & 43.5 & ND & ND & 46,8 & ND & ND & 145 & $2^{\circ}(46,7)$ & $-0,08$ & 148 & $3^{\circ}(52,6)$ & $-0,07$ \\
\hline 28 & $\mathrm{~F}$ & 11 anos e 9 meses & 54,0 & ND & ND & 61,8 & ND & ND & 147 & $2^{\circ}(43,5)$ & $-0,16$ & 150 & $2^{\circ}(49,1)$ & $-0,2$ \\
\hline 29 & M & 11 anos e 11 meses & 30,5 & ND & ND & 34.5 & ND & ND & 148 & $3^{\circ}(56,8)$ & 0,17 & 145 & $2^{\circ}(29,2)$ & $-0,55$ \\
\hline 18 & F & 12 anos e 2 meses & 52,0 & ND & ND & 59,0 & ND & ND & 155 & $3^{\circ}(74,9)$ & 0,67 & 157 & $3^{\circ}(74,9)$ & 0,70 \\
\hline 13 & $\mathrm{~F}$ & 13 anos e 11 meses & 58,4 & ND & ND & 61,5 & ND & ND & 154 & $2^{\circ}(24,4)$ & $-0,69$ & 159 & $2^{\circ}(45,6)$ & $-0,11$ \\
\hline
\end{tabular}

Pré, pré-operatório; Pós, pós-operatório. Idade expressa em anos e meses completos; peso, expresso em kg; altura,expressa em cm;Percetil e Z escore do desenvolvimento segundo a Organização Mundial de Saúde;ND, não definido 


\section{Discussão}

O anel de Waldeyer compreende um conjunto de estruturas linfoepiteliais que se dispõem em formato circular na cavidade oral, nasofaringe e rinofaringe e que possuem função de proteção imunológica, apresentando papel importante na defesa contra infecções. Seus componentes, classificados de acordo com sua localização, são: um par de tonsilas palatinas (também nomeadas de amigdalas palatinas ou amígdalas), a tonsila faringea (tonsila nasofaringea ou adenoide), um par de tonsilas tubárias, tonsilas linguais e pequenas coleções na parede da faringe. Essa distribuição na via de entrada do trato respiratório e alimentar reflete a sua finalidade, a de analisar e de reagir a antígenos provenientes do ar e da alimentação (1).

As amígdalas têm formato oval e são encontradas na fossa amigdaliana, entre os pilares amigdalianos anterior (músculo palatoglosso) e posterior (músculo palatofaringeo), e sobre o assoalho (músculo constritor superior da faringe). Apresentam cápsula e são recobertas por tecido epitelial estratificado escamoso, possuem inúmeras criptas, para o interior das quais o epitélio se invagina, de forma a aumentar a superfície com função imune (1, 3).

A tonsila faringea, por sua vez, é constituida por uma massa de tecido único situado no teto da rinofaringe. É revestida por epitélio estratificado pavimentoso e por epitélio colunar pseudoestratificado ciliado, sendo, assim, epitélio respiratório. Como as amigdalas, apresenta invaginações, porém, com dobras mais proeminentes, e uma cápsula fina (1, 3).

Ambas as tonsilas estão presentes já ao nascimento. Fisiologicamente aumentam durante a infância e involuem na adolescência com a formação de tecido fibrótico. Entretanto, de forma anormal, podem ocorrer crescimento intenso ou ausência da redução esperada proporcionando obstrução ao fluxo aéreo $(2,7,18)$.

A hipertrofia das tonsilas é a principal causa de obstrução de vias aéreas superiores em crianças, sendo capaz de proporcionar consequências como Sindrome da Apneia Obstrutiva do Sono, distúrbios de deglutição e fonação e amigdalites de repetição. Essas alterações possuem impacto no desenvolvimento pôndero-estatural das crianças. Outras possiveis consequências graves de são o desenvolvimento de cor pulmonale e o risco aumentado de morte súbita. Devido a essas condições, muitas crianças são submetidas à adenoamigdalectomia $(3,5,19)$.

Dados epidemiológicos mostram que no Reino Unido, por exemplo, são realizadas cerca de 200 mil adenoamigdalectomias anualmente; nos Estados Unidos da América são feitos mais de 500 mil procedimentos por ano, correspondendo a um terço de todas as cirurgias realizadas sob anestesia geral. No Brasil, a adenoamigdalectomia também se encontra entre os procedimentos mais realizados pelos otorrinolaringologistas: conforme dados do Data SUS sobre internações para realização de adenoamigdalectomia, no periodo de janeiro de 2018 a janeiro de 2019, foram realizadas 32.211 cirurgias apenas no âmbito do sistema público $(9,20,21,22,23)$.

O diagnóstico da hipertrofia das amígdalas pode ser realizado durante o exame físico otorrinolaringológico. A execução da oroscopia associada à escala de Brodsky pode indicar e classificar a presença do aumento destas tonsilas. Seguindo essa classificação temos: grau o, tonsilas situadas na fossa amigdaliana sem causar projeção na orofaringe; grau I, tonsilas levemente externas à fossa amigdaliana, com menos de 25\% de obstrução da via aérea; grau II, tonsilas prontamente visiveis, causando 25 a 50\% de obstrução; grau III, tonsilas ocupando 50 a $75 \%$ da via aérea; e grau IV, tonsilas com projeção significativa acima de $75 \%$, ocasionando obstrução da via aérea - sendo os graus III e IV mais prejudiciais ao fluxo de ar (18).

A avaliação do crescimento da tonsila faringea pode ser feita por meio de radiografia de cavum, que revela redução da coluna aérea da rinofaringe, ou por meio da fibronasoendoscopia, que permite a visualização direta de tal tonsila, sua mensuração e sua relação, por exemplo, com o óstio da tuba auditiva. De forma geral, considera-se uma tonsila faringea hipertrofiada quando ocupa área igual ou maior que $70 \%$ do cavum $(3,18)$. 
O tratamento de escolha em ambos os casos de hipertrofia com obstrução é a cirurgia, denominada adenoamigdalectomia, que compreende a remoção das duas tonsilas. Outros estudos comprovam que há benefício no padrão de crescimento após o procedimento médico conhecido como "estirão" pós-cirúrgico $(2,3,5,9,11)$.

A literatura aponta algumas explicações para a melhora do desenvolvimento pôndero-estatural das crianças após a retirada das amígdalas e da tonsila faringea. Dentre elas, pode-se citar uma melhora das funções mastigatória e deglutatória, decorrente da interferência causada pela hiperplasia adenoideana, que leva à diminuição do olfato, o que, em conjunto, pode originar diminuição do apetite e baixa ingestão calórica $(5,24)$.

Outra explicação possivvel decorre das consequências da apneia obstrutiva do sono, que cursa com baixa saturação de oxigênio e acidose, gerando aumento do trabalho respiratório e, consequentemente, aumento do gasto energético. Ainda, se percebem relatos indicam que essa situação, ao provocar despertares e microdespertares noturnos, ocasiona secreção irregular do hormônio do crescimento, normalmente liberado durante o sono na fase de ondas lentas, o que interfere no crescimento dessas crianças. Além disso, há indícios que após a adenoamigdalectomia ocorre aumento da secreção do hormônio do crescimento, do fator de crescimento semelhante à insulina e do fator de crescimento semelhante à insulina ligado à proteina transportadora B3. sendo estes dois últimos responsáveis pelo efeito anabólico do hormônio do crescimento $(5,7,12$, 25, 26, 27, 28, 29, 30).

Em relação à quantificação da velocidade de crescimento, a literatura aponta que o crescimento estatural normal é, em média, de sete centímetros por ano, ou seja, cerca de dois centímetros por trimestre, para crianças na faixa etária de três anos, até o início da puberdade. Outra forma de avaliação da velocidade de crescimento pode ser obtida ao realizar os cálculos propostos por Cole, que utiliza o escore $z$ da primeira (no dia da cirurgia) e da segunda medida (quatro meses depois da cirurgia) e, em seguida, realiza a subtração do primeiro em relação ao segundo valor obtido. Caso a diferença seja maior que zero, aponta-se uma velocidade em ascensão; se o valor encontrado for igual a zero, considera-se uma velocidade estável de crescimento; e, por último, se o valor obtido é menor que zero, a velocidade de crescimento é descendente. No presente estudo essa variação do escore $Z$ apresentou-se positiva para variável peso em 20 indivíduos entre os 22, uma vez que o peso para adolescentes não possui uma definição de referência pela Organização Mundial de Saúde. Ao considerar a altura, essa evidenciou correlação positiva em 21 indivíduos dentre os 29 participantes indicando ascensão na velocidade de crescimento $(13,13,32)$.

Ainda em relação à altura, percebe-se que na amostra, em média, houve aumento da altura em três centímetros, decorridos quatro meses do procedimento cirúrgico, que corresponde a uma velocidade de crescimento mais elevada que o esperado para a média da população. 0 desenvolvimento reforçado pós-cirúrgico já fora relatado por Everett como um crescimento acelerado compensatório ("catch-upgrowth") (12, 14, 28).

Além do crescimento em estatura pôde-se avaliar o desenvolvimento ao ponderar o ganho de peso anual das crianças; na faixa etária analisada, a literatura aponta como esperado o ganho de cerca de três quilos por ano. Em relação aos pacientes acompanhados, observou-se uma média de ganho de peso após a cirurgia, ao redor de 3,6 quilos, considerando os quatro meses de análise (17).

De forma complementar, notou-se que o IMC teve elevação em 20 participantes da pesquisa, o que corrobora com os achados de pesquisa em que esse fora a variável em análise, sendo explicado pela melhora em condições de ingestão alimentar, seja por desejo, seja por melhoras anatômicas para mastigação conforme apresentado anteriormente $(9,11)$

Ao analisar o ganho pôndero-estatural das crianças submetidas à adenoamigdalectomia, não foi possivel perceber aumento das medidas de peso e de estatura com significância estatística na 
avaliação dos grupos etários, apesar da melhora clínica e da variação percebida no escore Z e percentil de forma individual. Aspecto antagônico aos achados na literatura, evidenciados por Di Francesco, Fernandes, Williams e Schiffmann (5, $11,19,20,28,27)$.

O tamanho da amostra e o estado nutricional dos participantes da pesquisa podem ter influenciado para obtenção dos dados contrários à literatura. Individuos eutróficos e com classificação adequada de percentil e Z escores, ao serem submetidos à análise, apresentaram maior probabilidade de desenvolver resultados sem significância estatística.

É importante relatar, ainda, a dificuldade de seguimento pós-operatório dos pacientes por período prolongado, visto que após melhora importante do quadro clínico, os pais tendem a não retornar para reavaliações. Dessa forma, apesar de considerar quatro meses de pós-operatório um intervalo de tempo curto para alterações mais evidentes no padrão de ganho de peso e estatura, um resultado positivo já sinaliza a tendência à melhora dos parâmetros avaliados.

A partir da execução do estudo foi possivel observar que as crianças submetidas à adenoamigdalectomia, ao serem agrupadas, apresentaram ganho pôndero-estatural não estatisticamente significativo após a cirurgia, apesar da melhora clínica. Dessa forma, o diagnóstico da hipertrofia e da hiperplasia das amígdalas e da tonsila faríngea deve ser precoce e o tratamento instituido deve ser a cirurgia, quando formalmente indicada, a fim de evitar a manutenção do atraso no crescimento e, principalmente, em relação à melhora da Sindrome da Apneia Obstrutiva do Sono e às repercussões na fonação e na deglutição.

\section{Notas}

\section{Apoio financeiro}

Este estudo não recebeu apoio financeiro de fontes externas.

\section{Declaração de conflito de interesses}

Os autores declaram não haver conflitos de interesses relevantes ao conteúdo deste estudo.

\section{Contribuições dos autores}

Todos os autores fizeram contribuições substanciais para concepção, ou delineamento, ou aquisição, ou análise ou interpretação de dados; e redação do trabalho ou revisão crítica; e aprovação final da versão para publicação.

\section{Disponibilidade dos dados e responsabilidade pelos resultados}

Todos os autores declaram ter tido total acesso aos dados obtidos e assumem completa responsabilidade pela integridade destes resultados.

\section{Referências}

1. Marcelino TF, Silva ACB, Stüker PA, Leal VP, Anesi S, da Silva Júnior AF. Perfil dos pacientes submetidos à adenoamigdalectomia no Hospital Nossa Senhora da Conceição no ano de 2012-2013. Arq Catarin Med. 2014:43(4):30-33.

2. Wetmore RF. Surgical management of the tonsillectomy and tonsila faringeactomy patient. World J Otorhinolaryngol Head Neck Surg. 2017:3(3):176-82. https://dx.doi.org/10.1016\%2Fj.wjorl.2017.01.001

3. Marinho AF. Amigdalas e tonsila faringeas - da infecção à obstrução. Rev Port de Otorrinolaringol e Cirurgia Cérvico-Facial. 2010;48(1):25-32.

4. Godinho R, Cherobin G, Sih T. Crianças com Diferenças Faciais: O problema das Tonsilas Palatinas e da Tonsila faringea. In: Sih T, organizador. VIII Manual de Otorrinolaringologia Pediátrica da IAPO. São Paulo: Editora e Gráfica Vida \& Consciência; 2009. p. 74-81.

5. Di Francesco RC, Junqueira AP, Frizzarini R, Zerari FE. Crescimento pôndero-estatural de crianças após adenoamigdalectomia. Rev. Bras. Otorrinolaringolol. 2003;69(2):193-96. https://dx.doi.org/10.1590/S0034729.92003000200008

6. Simões APB, Palchetti CZ, Patin RV, Maurin JF, Oliveira FLC. Estado nutricional de crianças e adolescentes hospitalizados em enfermaria de cirurgia pediátrica. Rev Paul Pediatr. 2010;28(1):41-7. https://dx.doi.org/10.1590/ $\underline{\mathrm{S} 0103-05822010000100008}$

7. Filho AAB, Pereira FM, Minsky RC, Schivinski CIS. Adenoamigdalectomia E. O Crescimento Infantil - Segundo Marcadores Biológicos E Antropométricos. Arq Catarin Med. 2019:48(1):182-96.

8. Esteller E, Villatoro JC, Pedemonte G, Agüero A, Ademà JM, Girabent-Farrés M. Tratamiento quirúrgico del sindrome de apnea obstructiva Del sueño infantil: comparación entre adenoamigdalectomía extracapsular con bisturi frío y reducción mediante radiofrecuencia bipolar. Acta Otorrinolaringol Esp. 2016;67(5):261-7. https://doi.org/10.1016/j.otorri.2015.11.002 
9. Cavichiolo JB, Carvalho B, Alcântara LJL, Zimmermann E, Carvalho Filho S, Mocellin M. Perfil cirúrgico otorrinolaringológico em um hospital pediátrico de Curitiba. Arquivos Int Otorrinolaringol. 2010;14(4):422-5. https://dx.doi.org/10.1590/S1809-48722010000400007

10. Glover, JA. The Incidence of Tonsillectomy in Schoolchildren. Int J of Epidemiology. 2008;37(1):9-19. https:// doi.org/10.1093/ije/dym258

11. Fernandes AA, Alcantara TA, D'Avila DV, D'Avila JS Estudo das medidas pôndero-estaturais em crianças adenotonsilectomizadas. Rev. Bras. Otorrinolaringol. 2008;74(3):391-4. https://dx.doi.org/10.1590/So03472992008000300013

12. Tahara S, Hara H, Yamashita H. Evaluation of body growth in prepubertal Japanese children with obstructive sleep apnea after adenotonsillectomy over a long post operative period. Int J Pediatr Otorhinolaryngol. 2015;79(11):1806-9. https://doi.org/10.1016/j. ijporl.2015.08.005

13. BottiSho, Rego S. Processo ensino-aprendizagem na residência médica. Rev. bras. educ. med. 2010;34(1):132-140.

14. Skare TL. Metodologia do ensino na preceptoria da residência médica. Rev. Med. Res. 2012;4(2):116-20. https://dx.doi.org/10.1590/S0100-55022010000100016

15. Zeferino AM, Barros Filho AA, Bettiol H, Barbieri MA. Monitoring growth. J Pediatr (Rio J). 2003:79(Suppl 1):S23-32. https://dx.doi.org/10.1590/S002175572003000700004

16. Sociedade Brasileira de Pediatria. Manual de orientação para a alimentação do lactente, do pré-escolar, do escolar, do adolescente e na escola. Rio de Janeiro: Sociedade Brasileira de Pediatria; 2012. 148 p.

17. Windfuhr JP, Toepfner N, Steffen G, Waldfahrer $F$, Berner R. Clinical practice guideline: tonsillitis II. Surgical management. Eur Arch Otorhinolaryngol. 2016;273(4):989-1009. https://doi.org/10.1007/s00405016-3904-X

18. EverettAD, Koch WC, Saulsbury FT. Failure to thrive due to obstructive sleep apnea. Clin Pediatr. 1987;26(2):90-2. https://doi.org/10.1177/000992288702600206

19. Sciffmann R, Faber J, Eidelman Al. Obstructive hypertrophic adenoids and tonsils as a cause of infatile failure to thrive: reversed by tonsillectomy and tonsila faringe actomy. Int J Pediatr Otorhinolaryngol. 1985:9(2):183-7. https://doi.org/10.1016/s0165-5876(85)80019-7

20. Yilmaz MD, Hosal S, Oguz H, Yordam N, Kaya S. The effects of tonsillectomy adenoidectomy on serum IGF-I and IGFBP3 levels in children. Laryngoscope. 2002;112(5):922-5. https://doi.org/10.1097/00005537200205000-00026

21. American Academy of Otolaryngology - Head and Neck Surgery [Online]. Clinical Indicators: Tonsillectomy, Adenoidectomy, Adenotonsillectomy in Childhood. 2012 lacesso em 27 jul. 2019]. Disponivel em: https:// www.entnet.org/sites/default/files/TA-Adenotonsillectomy-Cl\%20Updated\%208-7-14.pdf
22. Bohr C, Shermetaro C. Tonsillectomy and Tonsilafaringe actomy. [Updated 2019 Jan 13]. In: Stat Pearls [Online]. Treasurelsland (FL): Stat Pearls Publishing; 2019 lacesso em 3 jul. 2019]. Disponivel em: https:// www.ncbi.nlm.nih.gov/books/NBK536942

23. Ministério da Saúde. DATASUS. AlH aprovadas segundo caráter atendimento procedimento: 0404010032 Amigdalectomia com Tonsilectomia Faríngea Periodo: Jan/2018-Jan/2019. [Online] 2019 lacesso em 3 jul. 2019]. Disponível em: http://tabnet.datasus.gov.br/ cgi/tabcgi.exe?sih/cnv/qgbr.def

24. Junqueira PAS, Di Francesco RC, Trezza P, Frizzarini R, Faria ME. Alterações funcionais do sistema estomatognático pré e pós-adenoamigdalectomia. Rev Pró-fono. 2002;4(1):17-22.

25. Su MS, Xu L, Pan WF, Li CC. Current perspectives on the correlation of nocturnal enuresis with obstructive sleep apnea in children. World J Pediatr. 2019;15(2):10916. https://doi.org/10.1007/s12519-018-0199-6

26. Baugh RF, Archer SM, Mitchell RB, Rosenfeld RM, Amin R, Burns JJ, et al. Clinical practice guideline: tonsillectomy in children. Otolaryngol Head Neck Surg. 2011;144(1 Suppl):S1-30. https://doi. org/10.1177/0194599810389949

27. Williams EF 3rd, Woo P, Miller R, Kellman RM. The effects of adenotonsillectomy on growth in young children. Otolaryngol Head \& Neck Surg. 1991;104(4):509-16. https://doi.org/10.1177/019459989110400415

28. Garcia-Garcia F, Juárez-Aguilar E, Santiago-García J, Cardinali DP. Ghreli and its interactions with growth hormone, leptin and orexins: Implications for the sleep-wake cycle and metabolism. Sleep Med Rev. 2014;18(1):8997. https://doi.org/10.1016/j.smrv.2013.04.003

29. Farmarzi M, Shishegar M, Heydari ST, Haghighi A, Sharouny $\mathrm{H}$. Effects of adenotonsillectomy on serum levels of IGF-1 and IGFBP-3 and growth indices in children with adenotonsillar hypertrophy or recurrent tonsillitis. Iran J Otorhinolaryngol. 2016;28(5):329-35.

\section{André Costa Pinto Ribeiro}

Mestrando em Saúde pela Faculdade de Medicina da Universidade Federal de Juiz de Fora (UFJF), em Juiz de Fora, MG, Brasil; vice-supervisor do Programa de Residência de Otorrinolaringologia do Hospital Universitário da UFJF, em Juiz de Fora, MG, Brasil.

\section{Tarssius Capelo Candido}

Mestrando em Saúde Coletiva pela Faculdade de Medicina da Universidade Federal de Juiz de Fora (UFJF), em Juiz de Fora, MG, Brasil. Preceptor Associado do Serviço de Otorrinolaringologia do Hospital Universitário da UFJF, em Juiz de Fora, MG, Brasil. 


\section{Pedro Henrique Almeida Nascimento}

Especialista em Otorrinolaringologia; egresso da turma 2020 do Serviço de Otorrinolaringologia do Hospital Universitário da Universidade Federal de Juiz de Fora (HU/UFJF), em Juiz de Fora, MG, Brasil.

\section{Paula Ferraz Rodrigues}

Médica R3 do Serviço de Otorrinolaringologia do Hospital Universitário da Universidade Federal de Juiz de Fora (HU/UFJF), em Juiz de Fora, MG, Brasil.

\section{Wilson Benini Guércio}

Mestre em Otorrinolaringologia pela Universidade Federal do Rio de Janeiro (UFRJ), no Rio de Janeiro, RJ, Brasil. Chefe do Serviço e Supervisor do Programa de Residência em Otorrinolaringologia do Hospital Universitário da Universidade Federal de Juiz de Fora (HU/UFJF), em Juiz de Fora, MG, Brasil.

\section{Endereço para correspondência}

André Costa Pinto Ribeiro

Hospital Universitário da Universidade Federal de Juiz de Fora

Unidade Dom Bosco

Av. Eugênio do Nascimento, s/n, $1^{\circ}$ andar, setor Métodos Gráficos

$36038-330$

Juiz de Fora, MG, Brasil 